\title{
EFFICIENT DOWNLOADING AND UPDATING APPLICATIONS ON PORTABLE DEVICES USING AUTHENTICATION TREES
}

\author{
Luke O'Connor* and Günter Karjoth \\ IBM Research \\ Zurich Research Laboratory \\ luke.oconnor@ch.unisys.com, gka@zurich.ibm.com
}

\begin{abstract}
Consider the problem of securely downloading $n$ application blocks $B_{1}, B_{2}, \ldots, B_{n}$ from an application provider to a smart card (SC) with the possibility of updating a block $B_{i}$ at a later time. In this paper we describe a scheme, which solves the problem of downloading and updating application blocks with the following properties: (1) a single signature based on an authentication tree is computed for the blocks, (2) only $O(\log n)$ additional memory is required by SC beyond the memory for the blocks themselves, (3) block $B_{i}$ can be verified as correct upon receipt at the $\mathrm{SC}$, and (4) blocks can be updated in $O(\log n)$ time. All previously known solutions require $O(n)$ memory and/or delay requirements for the download/update of $n$ code blocks. This scheme also generalizes to other portable devices that share common characteristics with smart cards.
\end{abstract}

Keywords: Portable device, smart card, authentication tree, digital signature.

\section{INTRODUCTION}

A general computing device such as a $\mathrm{PC}$ or a workstation stores applications in some permanent storage media, such as a hard disk, and then reads an application into main memory (RAM) for execution as required. Subject to available memory, new applications can be freely added while old applications can be easily removed or updated. However for a smart card (SC), given its limited memory capacity, applications are typically

* Since collaborating on this paper, Luke O'Connor has joined Unisys (Schweiz) AG. 
loaded into its ROM at the time of fabrication. This approach is convenient for the large scale production of SCs supporting one or a few fixed applications. But this approach is less suitable if the set of applications to be supported is expected to change, or if old applications need to be updated, or if the number of cards supporting a given application are not to be produced in large quantities.

A more flexible approach is to design the SC so that applications can be downloaded to the card as required. For example, in the ESPRIT project CASCADE [CASCADE, 1997], an SC was designed where the pre-installed software consists of a small boot kernel, libraries for basic I/O and cryptography, and a secure downloading mechanism, where other applications and systems code are downloaded securely to a FLASH memory (approximately $16 \mathrm{~KB}$ ) to the card. As an application may be quite large with respect to the amount of RAM or bandwidth available to the $\mathrm{SC}$, it is anticipated that an application will be partitioned into blocks $B_{1}, B_{2}, \ldots, B_{n}$ and each block will be downloaded from an Application Provider (AP) in a separate communication to the SC. Also, if an application is to be updated, then only those blocks that have been modified need be re-installed on the SC. We note that the blocks may represent either application code and/or application data. Using this scheme of block partitioning, there are two parameters of interest in evaluating a given solution with respect to downloading and updating blocks: delay requirements and memory requirements.

Delay Requirements. The downloading or updating of blocks should be 'on-the-fly' in the sense that blocks that are incorrect due to some error should be detected quickly to avoid wasting bandwidth and memory. If a block $B_{i}$ arrives at time $t$ but cannot be verified (e.g. by a hash check) until the arrival of block $B_{i+d}$ at time $t+d$, we say that the verification of $B_{i}$ is delayed for $d$ blocks. For a given scheme, we are interested in the maximum delay for block verification.

Memory Requirements. During block verification, storage is required for intermediate calculations and the caching of intermediate values that will be used for future blocks verifications. As memory on SCs is limited we seek solutions that minimize memory requirements.

For code downloading, the worst case delay and memory requirements are both $O(n)$. An $O(n)$ delay means that all blocks must be received before verification can begin, whereas $O(n)$ memory means that an additional linear amount of space beyond the storage for the $n$ blocks themselves is required. A protocol demonstrates a gain in efficiency if either the mem- 
ory or verification delay is reduced from $O(n)$ to possibly $O(\log n)$ or even $O(1)$. We say that block verification is 'on-the-fly' if the maximum delay is $O(1)$.

\subsection{RELATED WORK}

Our research has been partly motivated by the fact that little work has been done to address the code download/update problem for portable devices. According to its authors, the CASCADE project report [Dhem, 1998] describes the first protocols that considered these problems. The solution in this case was to produce a hash vector $H=\left(H_{1}, H_{2}, \ldots, H_{n+1}\right)$ of $(n+1)$ components such that $H_{n+1}$ is randomly chosen and $H_{i} \leftarrow$ $h\left(H_{i+1}, B_{i}\right)$ for some hash function $h(\cdot)$ such as SHA-1 [SHA, 1994]. The AP signs $H_{1}$, and then sends the following $n+2$ messages to the SC:

$$
\operatorname{Sign}\left(H_{1}\right),\left(H_{1}, B_{1}\right),\left(H_{2}, B_{2}\right), \ldots,\left(H_{n}, B_{n}\right), H_{n+1} \text {. }
$$

The SC first verifies the signature on $H_{1}$ and then proceeds to verify the hash chain used to form the hash vector. Owing to the form of the chain defined above, each block $B_{i}$ can be verified after the next pair $\left(H_{i+2}, B_{i+1}\right)$ has been received, yielding a constant delay of $O(1)$. However if a block $B_{i}$ is to be updated, then the hash chain must be recomputed from position $i$ forward due to the linear nature of the hash chain. This scheme will be denoted as 'CASCADE with hashes'. Hash chains have been widely used in a number of applications, such as one-time authentication [Lamport, 1981, Haller, 1994] or micro-payments [Hauser et al, 1996, Pedersen, 1996, Rivest and Shamir, 1996].

As noted by Dhem [Dhem, 1998], the $n$ hash values $H_{1}, H_{2}, \ldots, H_{n}$ need not be sent by the AP, because these values can be generated by the SC. We will refer to this scheme as 'CASCADE without hashes'. However the penalty for this reduced transmission is that the code block verification cannot begin until $H_{n+1}$ has been received, meaning that the maximum block delay before verification is $O(n)$ when no hashes are sent. Regardless of whether the hashes are sent at the time of download, they have been discarded by the time of update and thus incur an $O(n)$ update time for a block.

A problem related to efficient application downloading/updating is that of signing digital data streams. The solution that has commonalities with our results is the Wong-Lam scheme [Wong and Lam, 1999] based on authentication trees [Merkle, 1989]. Their protocol breaks a data stream into $n$ packets $P_{1}, P_{2}, \ldots, P_{i}, \ldots, P_{n}$ that are collected into a transmission group $T G$. The packets of $T G$ are then arranged to be the leaves of an authentication tree $T$, and the hash of the tree is computed and signed by the sender to produce $\operatorname{Sign}(T G)$. When $T G$ is transmit- 
ted, each packet $P_{i}$ is sent with the sequence of hash values that were used to form the path in the hash tree from the leaf representing $P_{i}$ to the root of the authentication tree $T$. The signature $\operatorname{Sign}(T G)$ on the authentication tree $T$ is also sent with each packet and packet hash path. This permits each packet $P_{i}$ to be verified as it is received, even though other packets in the $T G$ of $P_{i}$ may have been lost or reordered. To verify a given packet $P_{i}$, the receiver is typically required to recompute the path in the authentication tree from the leaf representing $P_{i}$ to the root of $T$, and then verify $\operatorname{Sign}(T G)$ based on the computed root hash. The full hash path from the leaf to the root must be computed for the first packet received, but the verification of subsequent packets can be optimized by reusing hash values that were previously computed, verified and then cached. The next received packet $P_{i+1}$ is verified by hashing it until a node in the cache is reached that was previously authenticated. The cache structure suggested by Wong-Lam mimics the structure of the original authentication tree that the sender used to compute the signature on $T G$. The receiver then requires a storage of the size $O(n)$ because this is the size of the authentication tree.

Authentication trees have also been used in a number of applications, for example micro-payments [Jutla and Yung, 1996].

\subsection{NEW CONTRIBUTIONS}

The solution to the application code download/update problem presented in this paper is called Ordered Tree Authentication, or simply OTA. OTA gives a $O(1)$ verification delay and logarithmic time for block update. This is achieved by sending a particular ordered sequence of hash values from the authentication tree along with the blocks to be authenticated, thus allowing verification of nodes of the tree besides the root. The OTA algorithm improves the approaches described in Section 1.1 in two aspects. First, the OTA algorithm needs a significantly lower amount of verification data to be transmitted with each data packet than WongLam. Second, the OTA algorithm lowers the amount of storage required at the receiving end without increasing the time for verification at the receiver. Thus, the OTA algorithm allows safe transmission between an application or service provider and a portable device having a limited storage or memory capacity and/or restricted processing power, such as smart cards and the like. A comparison between previous schemes and the proposed OTA method is given in Table 1.

The remainder of this paper is as follows. Section 2 begins with an example of an authentication tree that will be used to display the workings of OTA. The OTA scheme is described in Section 3, where Section 3.1 de- 
Table 1 Summary of time and storage requirements for block download and update.

\begin{tabular}{lcccc}
\hline \multicolumn{1}{c}{ Method } & \multicolumn{2}{c}{ Download } & \multicolumn{2}{c}{ Update } \\
& Storage & Max Delay & Storage & Max Delay \\
\hline CASCADE with hashes & $O(1)$ & $O(1)$ & $O(n)$ & $O(1)$ \\
CASCADE without hashes & $O(n)$ & $O(n)$ & $O(1)$ & $O(n)$ \\
Tree Authentication & $O(\log n)$ & $O(n)$ & $O(\log n)$ & $O(\log n)$ \\
Wong-Lam & $O(n)$ & $O(1)$ & N/A & N/A \\
OTA & $O(\log n)$ & $O(1)$ & $O(\log n)$ & $O(\log n)$ \\
\hline
\end{tabular}

tails the format of the blocks at the AP for transmission, and Section 3.2 details the process that the SC uses to verify the blocks. Section 4 shows how to deal with applications whose number of blocks is not a power of 2 , and discusses the problem of incrementing individual blocks. Conclusions are presented in Section 5.

\section{AUTHENTICATION TREES}

As orginally proposed by Merkle [Merkle, 1989], an authentication tree is a data structure used to authenticate individual data items such as the blocks $B_{1}, B_{2}, \ldots, B_{n}$. The basic idea is to select a labeled binary tree $T$ with $n=2^{d}$ leaves and to associate $B_{i}$ with the $i$-th leaf. For simplicity, we assume that $n$ is a power of two but this is not required for the construction. The length of the path from the root to a node $x$ is the depth of $x$ in $T$. The root is at depth 0 and there are $2^{i}$ nodes at depth $i$. An authentication tree $T$ has height $d$, the largest depth of any node in $T$. Tree $T$ has exactly $n$ leaves associated with the values of $B_{1}, B_{2}, \ldots, B_{n}$ and exactly $n-1$ internal nodes with two children each.

To compute the hash of the tree, the $i$-th leaf is labeled $H_{i}=h\left(B_{i}\right)$, where $B_{i}$ is associated with the leaf. Then, beginning at depth $d$ and proceeding to the root at depth 0 , each internal node $j$ is labeled

$$
H_{j}=h\left(L\left(H_{j}\right) \| R\left(H_{j}\right)\right)
$$

where $\|$ denotes concatenation and $L\left(H_{j}\right)$ and $R\left(H_{j}\right)$ are the labels of the left and right child, respectively, of node $j$. The label at the root, denoted $H_{T}$, is a hash value that depends on $B_{1}, B_{2}, \ldots, B_{n}$. The structure of an authentication tree on $n=8$ values $B_{1}, B_{2}, \ldots, B_{8}$ is shown in Figure 1 .

The AP signs the hash $H_{T}$ of $T$, then sends $H_{T}$, its signature, and the blocks $B_{1}, B_{2}, \ldots, B_{n}$. To verify the signature on the blocks, the SC must repeat all the hashing computations on $T$ to determine $H_{T}$. Note that no block can be rejected as corrupted until all blocks have 


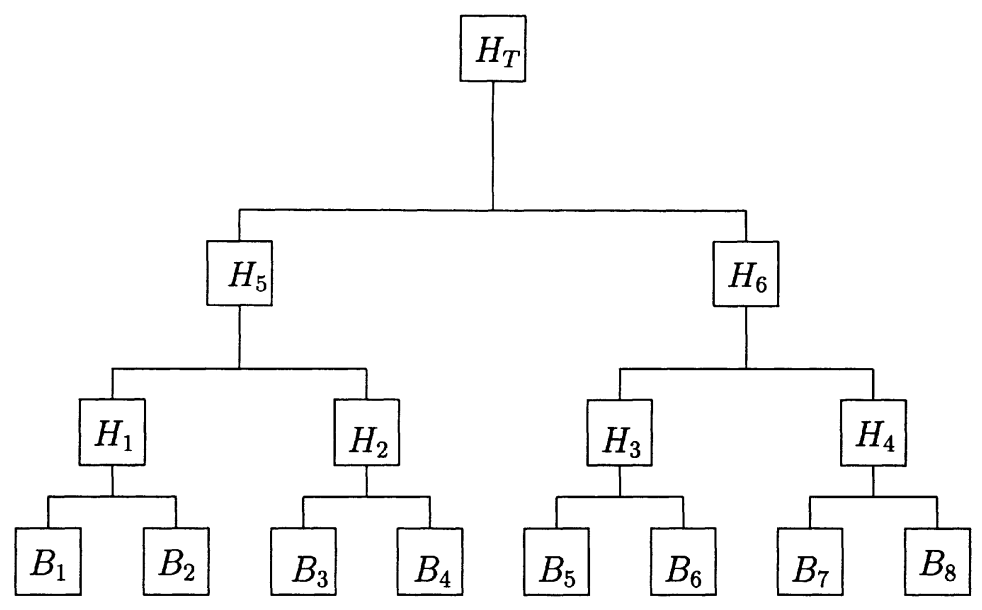

Figure 1 An authentication tree for $n=8$ and $d=3$.

been received, because the locally computed value of $H_{T}$ is not available until that time. Further, if the generated root hash does not match the received root hash, then the incorrect block(s) cannot be identified and all blocks must be retransmitted. Thus, the verification delay for basic tree authentication is $O(n)$. By recursively calculating the nodes as blocks are received, the verification of $T$ requires a memory of the size $O(\log n)$. Table 2 illustrates the verification of the authentication tree shown in Figure 1.

Table 2 Storage requirements to verify a tree authentication for $n=8$.

\begin{tabular}{lll}
\hline Received block & \multicolumn{1}{c}{ Compute } & Hash Storage \\
\hline$B_{1}$ & $h\left(B_{1}\right)$ & $h\left(B_{1}\right)$ \\
$B_{2}$ & $h\left(B_{2}\right), H_{1}$ & $H_{1}$ \\
$B_{3}$ & $h\left(B_{3}\right)$ & $H_{1}, h\left(B_{3}\right)$ \\
$B_{4}$ & $h\left(B_{4}\right), H_{2}, H_{5}$ & $H_{5}$ \\
$B_{5}$ & $h\left(B_{5}\right)$ & $H_{5}, h\left(B_{5}\right)$ \\
$B_{6}$ & $h\left(B_{6}\right), H_{3}$ & $H_{5}, H_{3}$ \\
$B_{7}$ & $h\left(B_{7}\right)$ & $H_{5}, H_{3}, h\left(B_{7}\right)$ \\
$B_{8}$ & $h\left(B_{8}\right), H_{4}, H_{6}$ & $H_{5}, H_{6}$ \\
$\operatorname{Sign}\left(H_{T}\right)$ & $H_{T}$ & $\operatorname{Sign}\left(H_{T}\right)$ \\
\hline
\end{tabular}


An advantage of the tree authentication over other methods such as hash chains is that an individual block can be updated in a logarithmic number of messages (or by a single message with a logarithmic number of components). To update $B_{i}$ to $B_{i}^{\prime}$, the AP first associates the $i$-th leaf with $B_{i}^{\prime}$ and then recomputes the hash values of the tree to yield the new root value $H_{T}^{\prime}$. The AP then signs the new root value $H_{T}^{\prime}$ and then sends $H_{T}^{\prime}$, its signature, and $B_{i}^{\prime}$. The $\mathrm{SC}$ then recomputes the hash tree of its blocks after replacing $B_{i}$ with $B_{i}^{\prime}$, and verifies that the newly computed root hash equals the received value of $H_{T}^{\prime}$. If the hashes agree, and the signature is correct, then $B_{i}$ is updated as $B_{i}^{\prime}$. Thus, using tree authentication, $n$ blocks can be downloaded in time $O(n)$ using $O(\log n)$ memory, and a block can be updated in $O(\log n)$ time and with $O(\log n)$ memory.

\section{ORDERED TREE AUTHENTICATION}

To verify a block, the verifier needs the tree signature and the siblings of each node in the block's path to the root. With this information, the verifier computes the hash values of each of the block's ancestors in the tree. That is, it first computes the hash value of the block, and then recursively the hash values of each ancestor by concatenating the last computed hash value with the corresponding node's sibling. For example, block $B_{3}$ is verified if $H_{2}^{\prime}=h\left(h\left(B_{3}\right) \| h\left(B_{4}\right)\right), H_{5}^{\prime}=h\left(H_{1} \| H_{2}^{\prime}\right), H_{T}^{\prime}=$ $h\left(H_{5}^{\prime} \| H_{6}\right)$, and $H_{T}=H_{T}^{\prime}$, where $H_{T}$ is contained in the tree signature.

Note that the above calculation also verifies nodes $H\left(B_{4}\right), H_{1}, H_{2}$, $H_{5}$, and $H_{6}$. If their values are cached, the verification of other blocks can be shortened. We define the last verified node for a block $B_{i}$ to be the closest verified ancestor on the block's path to the root of the authentication tree. For example, after the above calculation, block $B_{2}$ 's last verified node is $H_{1}$ and block $B_{4}$ 's last verified node is $H\left(B_{4}\right)$. In general, it is sufficient to verify a block against its last verified node.

In the OTA scheme, we exploit the above observations. By sending the tree signature first, the root becomes the last verified node for all blocks. Sending block $B_{1}$ and the siblings of each node in the block's path to the root, verifies not only the path from $H\left(B_{1}\right)$ to the root but also the siblings of the nodes on the path. We observe that removing the nodes on the path from leaf $H\left(B_{1}\right)$ to the root splits the authentication tree into $d$ subtrees, where $d$ is the height of the tree. The roots of the subtrees are the siblings of the nodes on the path. Each sibling is the last verified node of its children. In the OTA scheme, blocks are sent in sequence. Each message contains one block and all the nodes that constitute the block's path to its last verified node. 
In the remainder of this section, we specify the OTA scheme in detail via pseudo-code description of two routines called SendBlocks and ReceiveBlocks.

\subsection{SENDING BLOCKS}

As SendBlocks will act on the authentication tree $T$ for the blocks, we assume the following tree structure and operations based on the concept of a node. For our purposes a node has a parent, a left and a right child, and a hash value. Let function Parent $(\cdot)$ return the parent node and let function $\operatorname{Right}(\cdot)$ return the hash value of the right sibling. Let $H_{T}$ denote the hash of the authentication tree. As by construction the leaves of the authentication tree correspond to blocks, we also let $\operatorname{Node}\left(B_{i}\right)$ denote the leaf corresponding to $B_{i}$.

The pseudo-code for the SendBlocks routine is shown in Algorithm 3.1. This routine will be run by the AP that wishes to send the blocks $B_{1}, B_{2}, \ldots, B_{n}$ to the SC. Each code block $B_{i}$ will be sent in a message $M_{i}$, where $M_{i}$ will contain $B_{i}$ and possibly some additional hash values from $T$.

When the SendBlocks routine is executed, all $n$ blocks $B_{1}, B_{2}, \ldots, B_{n}$ are passed as inputs. Routine SendBlocks maintains the following variables: depth holds the height of the corresponding authentication tree $T$ of the blocks, cache is a boolean array of size depth +1 , and node is a pointer into tree $T$. A field cache[i] indicates whether a node on depth $i$ constitutes the verified root of a subtree for a block not yet received.

First, SendBlocks constructs and hashes the authentication tree $T$ for the blocks, with the resulting signature $\operatorname{Sign}\left(H_{T}\right)$ sent to the SC. Also cache $[0]$ is set to true to indicate that the root hash has been sent. Next, the messages corresponding to the blocks that will be used to verify this signature are created. Routine SendBlocks has a main for loop (steps 6 to 18) that executes $n$ times and processes $B_{i}$ at the $i$-th iteration to generate $M_{i}$, which is sent to the receiver at step 16 . At each iteration, message $M_{i}$ is initialized to $B_{i}$ (step 7), variable node points to $\operatorname{Node}\left(B_{i}\right)$, the leaf node corresponding to $B_{i}$ (step 8 ), and auxiliary variable $j$ is set to the depth of the leaf nodes of $T$ (step 9 ). Further processing adds any additional hash values to $M_{i}$ that will be required at the SC to perform the verification of $B_{i}$ after it is received.

We now explain how the additional hashes for $B_{i}$ are determined. The while loop from steps 10 to 15 traverses the path from $\operatorname{Node}\left(B_{i}\right)$ to the last verified node towards the root of the authentication tree by repeatedly assigning node to its parent (step 13). At each new node referenced by node for which the hash value of the right sibling has not been sent as 


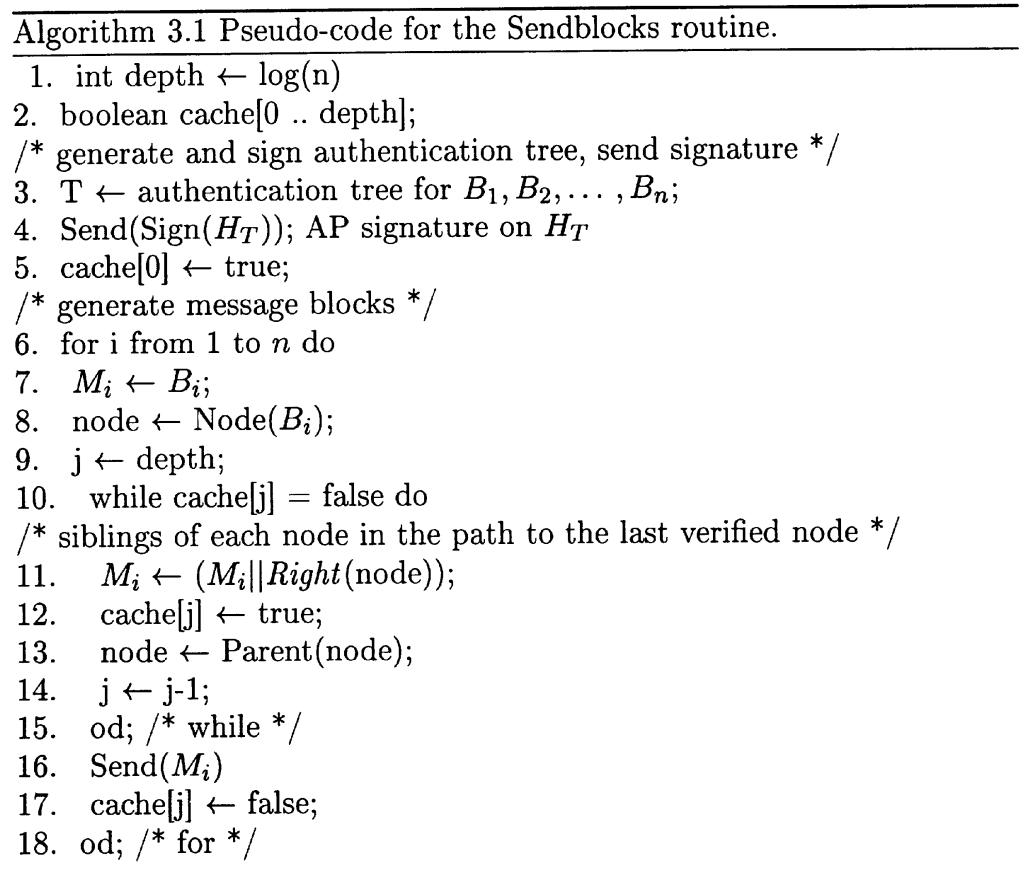

part of a previous message (that is $M_{1}, M_{2}, \ldots, M_{i-1}$ ), this hash value is appended to $M_{i}$ (step 11) and the cache field of the corresponding depth is set to true (step 12). Note that the while loop is executed for odd-numbered messages only; therefore there is always a right sibling. Equivalently, if cache $[j]$ is false, then $\operatorname{Right}\left(\right.$ node) is appended to $M_{i}$ at step 11 , and cache $[j]$ is set to true to indicate that this hash value need not be sent with any future message. This process of appending hash values to $M_{i}$ continues in the while loop until cache $[j]=$ true indicates that the hash value of this depth was sent by a previous message. At this point, the message $M_{i}$ for block $B_{i}$ is complete and can be sent to the SC. The creation of the next message $M_{i+1}$ for the block $B_{i+1}$ begins at the $(i+1)$-st iteration of the main loop of SendBlocks. The main loop continues in this manner until all $n$ messages for all $n$ blocks have been generated and sent, at which point SendBlocks exits.

Example 3.1 Consider executing SendBlocks on the authentication tree of Figure 1 with the blocks $B_{1}, B_{2}, \ldots, B_{8}$. After the signature on $H_{T}$ has been sent, the contents of the eight messages $M_{1}, M_{2}, \ldots, M_{8}$ corre- 
sponding to the blocks $B_{1}, B_{2}, \ldots, B_{8}$ are

$$
\begin{aligned}
& M_{1}=\left\{B_{1}, h\left(B_{2}\right), H_{2}, H_{6}\right\} \\
& M_{2}=\left\{B_{2}\right\} \\
& M_{3}=\left\{B_{3}, h\left(B_{4}\right)\right\} \\
& M_{4}=\left\{B_{4}\right\} \\
& M_{5}=\left\{B_{5}, h\left(B_{6}\right), H_{4}\right\} \\
& M_{6}=\left\{B_{6}\right\} \\
& M_{7}=\left\{B_{7}, h\left(B_{8}\right)\right\} \\
& M_{8}=\left\{B_{8}\right\}
\end{aligned}
$$

We note that the longest message is $M_{1}$ and that even-indexed messages contain no hash values because $h\left(B_{2 i}\right)$ will be sent with $M_{2 i-1}$.

We first prove a crucial property of the SendBlocks routine, the proper termination of the while loop.

Lemma 1 Before processing message $M_{i}, 1 \leq i \leq n$, there is at least one field in the cache set to true.

Proof. Before processing message $M_{1}$, cache[0] = true holds (step 5). At every iteration of the for loop, only the field with the highest index set to true is set to false (step 17). However, the inner while loop will flip all fields holding value false, starting from cache[depth] up to the field that corresponds to the depth of the last verified node. This means that all fields of the cache are only set to false if cache $[$ depth $]$ is the only true field (the while loop is not executed).

In other words, data structure cache behaves like a counter if we regard its fields to represent a binary number, where true denotes 1 and cache[0] is the most significant bit. Its initial value is $n=2^{\text {depth }}$. The for loop decrements its value at every iteration. After $n$ iterations the counter is zero.

Lemma 2 Message $M_{i}$ contains exactly those hash values that are required to compute the hash of a subtree of $T$ for which node $H\left(B_{i}\right)$ is the leftmost child.

Proof. To compute the above subtrees, it is sufficient that message $M_{i}$ contains block $B_{i}$ together with the siblings of each node in the path from leaf $H\left(B_{i}\right)$ to block $B_{i}$ 's last verified node. The proof is by induction.

Base case. Before creating message $M_{1}$, cache $[0]=$ true holds (step 5). Execution of the while loop adds to message $M_{1}$ the siblings of each node in block $B_{1}$ 's path to its last verified node, root $H_{T}$. In addition, 
fields cache[depth] to cache[1] are set to true, and field cache[0] is set to false.

Induction step. Let us assume that message $M_{i}$ has been sent. Case analysis.

$1 M_{i+1}$ is an even-indexed message: Then message $M_{i}$ carried the hash value of block $B_{i+1}$, the sibling of block $B_{i}$, and field cache $[$ depth $]=$ true. Thus, no hash value is added to message $M_{i+1}$ but cache $[$ depth $]=$ false holds afterwards.

$2 M_{i+1}$ is an odd-indexed message: Then block $B_{i+1}$ is the left child of its ancestor and its last verified node is at depth $j, 0<j<$ depth. Thus, execution of the while loop adds to message $M_{i+1}$ the siblings of each node in block $B_{i+1}$ 's path to its last verified node at depth $j$. In addition, fields cache[depth] to $c a c h e[j+1]$ are set to true, and field cache $[j]$ is set to false.

We now prove some simple properties concerning the messages generated by SendBlocks.

Lemma 3 Let $B_{1}, B_{2}, \ldots, B_{n}$ be a set of $n=2^{d}$ blocks and $M_{1}, M_{2}, \ldots, M_{n}$ the corresponding $n$ messages generated by SendBlocks for these $n$ blocks. Then no message $M_{i}$ contains more than $d$ hash values, and no more than $n-1$ hash values are sent in total with the $n$ messages.

Proof. The while loop of SendBlocks cannot execute more than $d$ times before the root of the authentication tree is reached, which proves the first statement of the lemma. To prove the second statement we observe that hash values appended to messages by SendBlocks must be labels of right children in the authentication tree. As there are $2 n-1$ nodes in the authentication tree, at most $n-1$ hashes of right children can be sent in messages.

The proof of this lemma shows that on average each message generated by SendBlocks contains at most one additional hash value.

\subsection{RECEIVING BLOCKS}

The ReceiveBlocks routine executes at the $\mathrm{SC}$ and verifies code blocks by processing the messages generated by the SendBlocks routine at the AP. The pseudo-code for the ReceiveBlocks routine is shown in Algorithm 3.2. ReceiveBlocks maintains a data structure called cache, which 
is an array of hash values. The fields of cache can be addressed from 0 up to $d$, the depth of the authentication tree $T$. For each depth $i$ of the authentication tree $T$, cache $[i]$ holds hash value $H_{j}$ of a node that was verified last in the processing of a previous message. In the setup phase (steps 2-5 of Algorithm 3.2), the ReceiveBlocks routine first reads and verifies the signature $\operatorname{Sign}\left(H_{T}\right)$ on the authentication tree $T$, extracts the hash value $H_{T}$ of the root, and then stores the hash $H_{T}$ of the authentication tree in field cache[0].

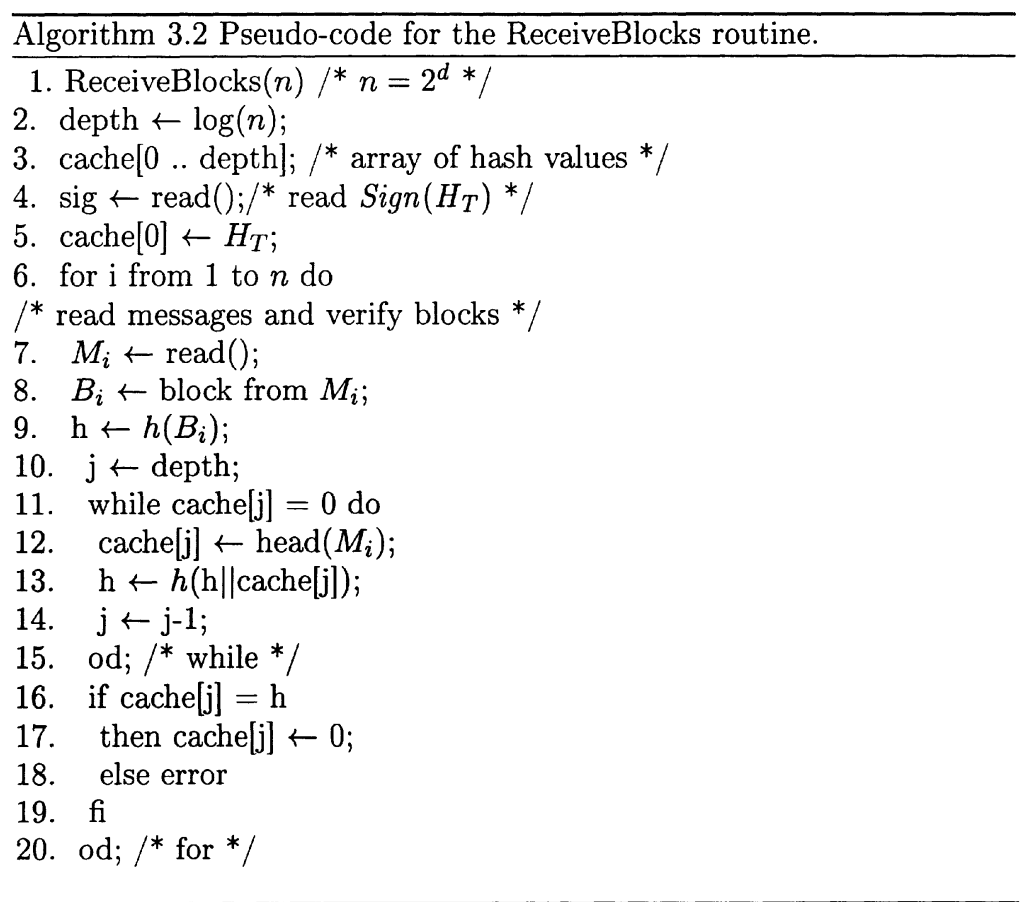

ReceiveBlocks has a main for loop (steps 6-20) that executes $n$ times, receiving and verifying the $i$-th block $B_{i}$ at the $i$-th iteration. At the $i$-th iteration, it receives message $M_{i}$ (step 7), extracts block $B_{i}$ from $M_{i}$ (step 8), computes the hash value $h\left(B_{i}\right)$ of that block, and stores the computed hash value in the temporary variable $h$ (step 9$)$. Next ReceiveBlocks simulates the computation of the hash path for $B_{i}$ in $T$ until it reaches a node which has already been verified in the processing of a previous message $M_{1}, M_{2}, \ldots, M_{i-1}$ (steps 10 - 15). For each not yet verified node, ReceiveBlocks extracts the hash value of its right sibling 
from the received message $M_{i}$ and stores the value in the corresponding field of the cache (step 12), computes the hash value of the parent node, and stores it in temporary variable $h$ (step 13). Finally, the routine compares the computed hash value stored in variable $h$ with the hash value of the already verified intermediate node (step 16). If the values are equal, block $B_{i}$ is considered verified and ReceiveBlocks clears the hash value of the already verified intermediate node in cache (step 17). Otherwise, ReceiveBlocks indicates an error (step 18). The main for loop continues in this manner until all $n$ messages for all $n$ blocks have been received and verified, at which point ReceiveBlocks exits.

Table 3 Storage requirements for OTA for $n=8$.

\begin{tabular}{llll}
\hline \multicolumn{1}{c}{ Received message } & \multicolumn{1}{c}{ SC Compute } & SC Verify & SC Hash Storage \\
\hline $\operatorname{Sign}\left(H_{T}\right)$ & - & - & $H_{T}$ \\
$\left\{B_{1}, h\left(B_{2}\right), H_{2}, H_{6}\right\}$ & $h\left(B_{1}\right), H_{1}, H_{5}, H_{T}$ & $H_{T}$ & $h\left(B_{2}\right), H_{2}, H_{6}$ \\
$\left\{B_{2}\right)$ & $h\left(B_{2}\right)$ & $h\left(B_{2}\right)$ & $H_{2}, H_{6}$ \\
$\left\{B_{3}, h\left(B_{4}\right)\right\}$ & $h\left(B_{3}\right), H_{2}$ & $H_{2}$ & $h\left(B_{4}\right), H_{6}$ \\
$\left\{B_{4}\right\}$ & $h\left(B_{4}\right)$ & $h\left(B_{4}\right)$ & $H_{6}$ \\
$\left\{B_{5}, h\left(B_{6}\right), H_{4}\right\}$ & $h\left(B_{5}\right), H_{3}, H_{6}$ & $H_{6}$ & $h\left(B_{6}\right), H_{4}$ \\
$\left\{B_{6}\right\}$ & $h\left(B_{6}\right)$ & $h\left(B_{6}\right)$ & $H_{4}$ \\
$\left\{B_{7}\right\}, h\left(B_{8}\right)$ & $h\left(B_{7}\right), H_{6}$ & $H_{4}$ & $h\left(B_{8}\right)$ \\
$\left\{B_{8}\right\}$ & $h\left(B_{8}\right)$ & $h\left(B_{8}\right)$ & - \\
\hline
\end{tabular}

Example 3.2 The operation of the ReceiveBlocks routine as run on the messages produced by SendBlocks from Example 3.1 is shown in Table 3. After the signature on $H_{T}$ has been verified, field cache[0] holds $H_{T}$. All other fields are empty. Next, ReceiveBlocks receives the first message: $M_{1}=\left\{B_{1}, h\left(B_{2}\right), H_{2}, H_{6}\right\}$. As the fields cache[3], cache[2], and cache[1] are empty, the while loop extracts the three hash values $h\left(B_{2}\right), H_{2}$, and $H_{6}$ from the message. These hash values are the siblings of the nodes on the path for $B_{1}$ and thus allow node $H_{T}$ to be computed and verified. As a side effect, nodes $h\left(B_{2}\right), H_{2}$, and $H_{6}$ are also verified and thus stored in the cache. As node $H_{T}$ is verified, field cache[0] is cleared.

Let us assume that the next message received contains an even-indexed block. Then the previous message contained the block's hash value and was stored in field cache[3]. Thus, the even-indexed block can be immediately verified and field cache[3] will be cleared. In general, whenever a node is verified, the corresponding field in the cache is cleared (step 17).

Finally, let us consider the case where the next message received contains an odd-indexed block $B_{2 j+1}$. We know from the discussion above that the previous iteration (iteration $2 j$ ) of the for loop cleared field cache[3] and thus at least the hash value of the next block $B_{2 j+2}$ will 
be extracted from the message. If the parent node has not been verified previously, the hash value of the parent's right child will be extracted from the message. Note that intermediate nodes are verified before their right child are verified. Thus, walking up towards the root until an already verified node is reached, the shortest hash path to verify the code block is obtained.

Lemma 4 The ReceiveBlocks routine verifies $B_{i}$ given $M_{1}, M_{2}, \ldots, M_{i}$ in constant time using $O(\log n)$ storage.

Proof. The proof is by induction.

Base case. Message $M_{1}$ carries the right siblings of each node in the path from $\operatorname{Node}\left(B_{1}\right)$ to but excluding the root $H_{T}$ (Lemma 2) that has been sent before and is kept in field cache[0]. With this information, SC can compute the root value of $T$ by iteratively computing the hash value of each parent node by combining the left child (first by computing the hash value of block $B_{1}$; later using the result of the last computation) with the hash value of the right child contained in message $M_{1}$. Block $B_{1}$ in message $M_{1}$ is verified. In addition, fields cache[depth] to cache[1] keep the hash values of the siblings, and field cache[0] is set to zero.

Induction step. Let us assume that blocks $B_{1}, B_{2}, \ldots, B_{i}$ have been verified. Consider verifying block $B_{i+1}$ with message $M_{i+1}$. Case analysis.

$1 M_{i+1}$ is an even-indexed message: According to Lemma 2, message $M_{i}$ carries the hash value of block $B_{i+1}$, the (right) sibling of block $B_{i}$. Hash value $h\left(B_{i+1}\right)$ was stored in field cache [ depth]. Thus, block $B_{i+1}$ can immediately be verified. Field cache $[$ depth] is set to zero.

$2 M_{i+1}$ is an odd-indexed message: Then block $B_{i+1}$ is the left child of its ancestor and its last verified node is at depth $j, 0<j<$ depth. Thus, message $M_{i+1}$ contains the siblings of each node in block $B_{i+1}$ 's path to its last verified node at depth $j$. Execution of the while loop calculates the hash value of block $B_{i+1}$ 's last verified node and compares that value with the hash value stored at field cache $[j]$. In addition, fields cache[depth] to cache $[j+1]$ keep the hash values of the siblings, and field cache $[j]$ is set to zero.

Routine ReceiveBlocks stores the intermediate hash values in array cache of size $\log n+1$. 


\section{EXTENSIONS}

\subsection{ARBITRARY NUMBER OF BLOCKS}

If the number of blocks to be downloaded is not a power of 2 , there is a simple change to adapt the two routines. Before executing the while loop, variable $\mathrm{j}$ is assigned the value of the height of the authentication tree $(\mathrm{j} \leftarrow$ depth). It is sufficient to replace the constant depth by a function, Depth $(\cdot)$, that returns the depth of the leaf associated with the current block to be processed. If we take, for example, an application that consists of three blocks, we get the authentication tree in Figure 2. The major difference is in sending/receiving the third block. After the second message has been processed, cache $[1]=h\left(B_{3}\right)$ holds. Setting the initial value of variable $j$ to $1\left(\mathrm{j} \leftarrow \operatorname{Depth}\left(B_{3}\right)\right.$ instead to $2(\mathrm{j} \leftarrow \operatorname{depth})$ skips the processing of the (missing) nodes at depth 2 .

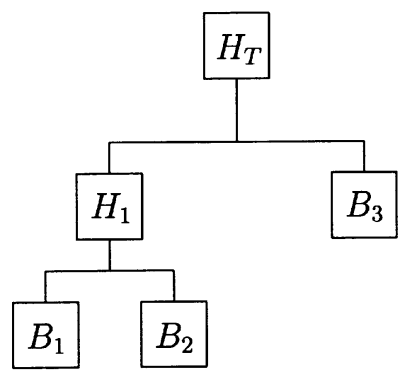

\begin{tabular}{ll}
\hline \multicolumn{1}{c}{ Message } & \multicolumn{1}{c}{ SC Storage } \\
\hline $\operatorname{Sign}\left(H_{T}\right)$ & $H_{T}$ \\
$\left\{B_{1}, h\left(B_{2}\right), h\left(B_{3}\right)\right\}$ & $h\left(B_{2}\right), h\left(B_{3}\right)$ \\
$\left\{B_{2}\right\}$ & $h\left(B_{3}\right)$ \\
$\left\{B_{3}\right\}$ & - \\
\hline
\end{tabular}

Figure 2 An authentication tree for $n=3$ and $d=2$.

\subsection{INCREMENTAL BLOCKS}

To add the new $B_{i+1}$ block to those existing in the SC, the AP adds $B_{n+1}$ to the $H_{T}$ as described above and then computes the new root value $H_{T}^{\prime}$. Then, the AP sends $H_{T}^{\prime}$, its signature $\operatorname{Sign}\left(H_{T}^{\prime}\right)$, the index $i$, and $B_{n+1}$ to the SC, where $i$ indicates the leaf in the current authentication tree that will become the parent of the new block. The SC inserts $B_{n+1}$ into the authentication tree, recomputes the hash tree and verifies that the newly computed root hash equals the received value of $H_{T}^{\prime}$. If the hashes agree, and the signature is correct, then $B_{n+1}$ is added to the blocks. The above protocol naturally extends to the case where $m$ new blocks are added.

It may also be the case that a given application of $n$ blocks $B_{1}, B_{2}, \ldots, B_{n}$ is to be increased to have $n+1$ blocks with the addition of a new block $B_{i+1}$. Adding a new block can be considered a special 
update operation in OTA. Let $H_{T}$ be the OTA authentication tree for the blocks $B_{1}, B_{2}, \ldots, B_{n}$, which will consist of a binary tree with internal nodes and leaves, where each internal node will have two children (excluding the case of $n=1$ ). Each leaf is a block $B_{i}$, and is positioned at some depth $d$ in $H_{T}$. To add a new block $B_{n+1}$ to $H_{T}$, one considers the set of leaves that are at the minimum depth $d$ in $H_{T}$, and pick one at random, here denoted $B_{i}$. Then the node for $B_{i}$ is replaced by an internal node that has $B_{i}$ and $B_{n+1}$ as its children.

\section{CONCLUSIONS}

The OTA scheme presented for downloading to and/or updating and authenticating data or applications on a portable device has clear advantages for the chosen specific example of a smart card as portable device as well as for other applications. The algorithm can easily be adapted and applied to any problem where complex applications must be downloaded to or updated in a device having constraints in memory, processing speed and/or bandwidth or where updating time and/or security play significant roles.

\section{References}

[CASCADE, 1997] See

http://www.dice.ucl.ac.be/crypto/cascade/cascade.html.

[SHA, 1994] (1994). FIPS 180-1, Secure Hash Standard, Federal Information Processing Standards Publication 185, US Department of Commerce/NIST, National Technical Information Service, Springfield, Virginia, 1994. Available at http://csrc.nist.gov/fips/fip180-1.ps.

[Dhem, 1998] Dhem, J.F. (1998). Design of an efficient public key cryptographic library for RISC-based smart cards. PhD thesis, Université catholique de Louvain. Available at http://www.dice.ucl.ac.be/crypto/dhem/dhem.html.

[Hauser et al, 1996] Hauser, R., Steiner, M., and Waidner, M. (1996). Micro-Payments based on iKP. 14th Worldwide Congress on Computer and Communications Security Protection, pages 67-82.

[Haller, 1994] Haller, N.M. (1994) The S/Key One-Time Password System. Internet Society Symposium on Network and Distributed Systems Security, pages 151-157.

[Jutla and Yung, 1996] Jutla, C. and Yung, M. (1996). PayTree: "Amortized-Signature" for Flexible MicroPayments. 2nd USENIX Workshop on Electronic Commerce, pages 213-222. 
[Lamport, 1981] Lamport, L. (1981). Password Authentication with Insecure Communication. Communications of the ACM, 24(11):770-772.

[Merkle, 1989] Merkle, R.C. (1989). A certified digital signature. Advances in Cryptology, CRYPTO 89, Lecture Notes in Computer Science 218, G. Brassard ed., Springer, pages 218-238.

[Pedersen, 1996] Pedersen, T.P. (1996). Electronic payments of small amounts. In Cambridge Workshop on Security Protocols, Lecture Notes in Computer Science 1189, Springer, pages 59-68.

[Rivest and Shamir, 1996] Rivest, R.L. and Shamir A., 1996. PayWord and MicroMint: Two simple micropayment schemes. In Cambridge Workshop on Security Protocols, Lecture Notes in Computer Science 1189, Springer, pages 69-88.

[Wong and Lam, 1999] Wong, C.K. and Lam, S.S. (1999). Digital signatures for flows and multicasts. IEEE/ACM Transactions on Networking, 7(4):502-513. 